Max-Planck-Institut für demografische Forschung

Max Planck Institute for Demographic Research

Konrad-Zuse-Strasse 1 - D-18057 Rostock · GERMANY

Tel +49 (0) 3812081 - 0; Fax +49 (0) 3812081 - 202;

http://www.demogr.mpg.de

MPIDR TECHNICAL REPORT 2012-001

APRIL 2012

\title{
Reshaping of Human Fertility Database data from long to wide format in Excel
}

Vladimir M. Shkolnikov (shkolnikov@demogr.mpg.de)

Dmitry A. Jdanov (jdanov@demogr.mpg.de)

For additional material see www.demogr.mpg.de/tr/

(C) Copyright is held by the authors.

Technical reports of the Max Planck Institute for Demographic Research receive only limited review. Views or opinions expressed in technical reports are attributable to the authors and do not necessarily reflect those of the Institute. 


\title{
Reshaping of Human Fertility Database data from long to wide format in Excel
}

\author{
by Vladimir M. Shkolnikov and Dmitry A. Jdanov
}

\section{Abstract}

In the Human Fertility Database (HFD, 2012), age-specific data are presented in the long format, with both the years (or the years of the mothers' births) and the mothers' ages at birth in descending order, and with the age-specific fertility rates (or birth counts) corresponding to various combinations of years and ages placed in one long column. For constructing figures and performing other actions in MS Excel, it is useful to have the same data in wide format with the years (or the years of the mothers' births) descending rightwards, and with age-specific fertility rates for these years placed in separate columns arranged next to each other. We provide a simple VBA/Excel program that reshapes long-format age-specific HFD data into a wide format.

Keywords: age-specific fertility rates, long format, wide format, reshape

\section{Background}

For each country, the Human Fertility Database (HFD) provides on the page entitled "Age-Specific Data" cohort and period data on birth counts, female population exposure, and age-specific and cumulative fertility rates (Figure 1). These data are most useful for users who perform their own analyses of fertility trends and age patterns.

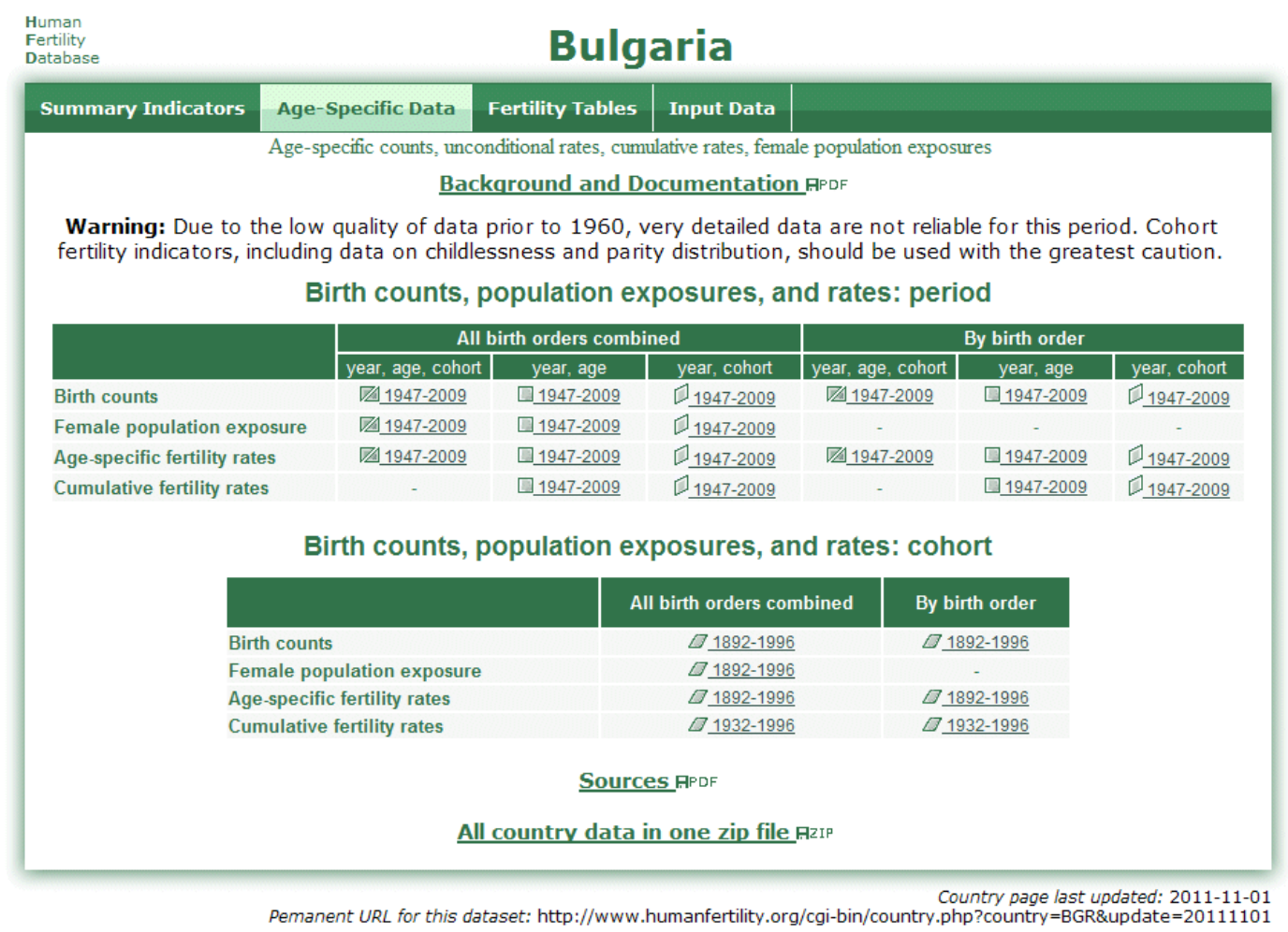

Figure 1. The HFD “Age-Specific Data” page for Bulgaria.

All of the datasets available on the Age-Specific Data page have the same long format. In them, the data elements (e.g., age-specific rates or birth counts) for all of the calendar years or years of the mothers' births are placed in one column. As an example, Figure 2 shows fragment of long-format 
ASCII file with period fertility rates by calendar year and the age of the mothers for Bulgaria.

\begin{tabular}{|c|c|c|}
\hline Year & age & $\mathrm{ASFR}$ \\
\hline 1947 & $12-$ & 0.00000 \\
\hline 1947 & 13 & 0.00009 \\
\hline 1947 & 14 & 0.00148 \\
\hline 1947 & 15 & 0.00579 \\
\hline 1947 & 15 & 0.01747 \\
\hline 1947 & 17 & 0.03915 \\
\hline 1947 & 18 & 0.05974 \\
\hline 1947 & 19 & 0.10710 \\
\hline 1947 & 20 & 0.14818 \\
\hline 1947 & 21 & 0.17945 \\
\hline 1947 & 22 & 0.19836 \\
\hline 1947 & 23 & 0.20587 \\
\hline 1947 & 24 & 0.21029 \\
\hline 1947 & 25 & 0.19504 \\
\hline 1947 & 25 & 0.17450 \\
\hline 1947 & 27 & 0.15670 \\
\hline 1947 & 28 & 0.14374 \\
\hline 1947 & 29 & 0.13567 \\
\hline 1947 & 30 & 0.12945 \\
\hline 1947 & 31 & 0.11090 \\
\hline 1947 & 32 & 0.09723 \\
\hline 1947 & 33 & 0.08711 \\
\hline 1947 & 34 & 0.07915 \\
\hline 1947 & 35 & 0.05818 \\
\hline 1947 & 35 & 0.05814 \\
\hline 1947 & 37 & 0.04814 \\
\hline 1947 & 38 & 0.03884 \\
\hline 1947 & 39 & 0.03054 \\
\hline 1947 & 40 & 0.02275 \\
\hline 1947 & 41 & 0.01720 \\
\hline 1947 & 42 & 0.01374 \\
\hline 1947 & 43 & 0.01129 \\
\hline 1947 & 44 & 0.00922 \\
\hline 1947 & 45 & 0.00703 \\
\hline 1947 & 45 & 0.00515 \\
\hline 1947 & 47 & 0.00277 \\
\hline 1947 & 48 & 0.00123 \\
\hline 1947 & 49 & 0.00048 \\
\hline 1947 & 50 & 0.00018 \\
\hline 1947 & 51 & 0.00007 \\
\hline 1947 & 52 & 0.00003 \\
\hline 1947 & 53 & 0.00001 \\
\hline 1947 & 54 & 0.00000 \\
\hline 1947 & $55+$ & 0.00000 \\
\hline 1948 & $12-$ & 0.00000 \\
\hline 1948 & 13 & 0.00010 \\
\hline 1948 & 14 & 0.00155 \\
\hline 1948 & 15 & 0.00525 \\
\hline 1948 & 16 & 0.01852 \\
\hline 1948 & 17 & 0.04084 \\
\hline 1948 & 18 & 0.07210 \\
\hline 1948 & 19 & 0.11121 \\
\hline 1948 & 20 & 0.15428 \\
\hline 1948 & 21 & 0.18830 \\
\hline 1948 & 22 & 0.20854 \\
\hline 1948 & 23 & 0.21582 \\
\hline 1948 & 24 & 0.21511 \\
\hline
\end{tabular}

Figure 2. Period age-specific fertility rates by calendar year and age of the mothers. A fragment of HFD data for Bulgaria.

In this file, the calendar years and ages are given in the first two columns, and the age-specific fertility rates (ASFRs) for every combination of calendar year and age are given the last column. The file begins from the ASFR values in 1947 for ages 12 and younger, 13, 14, .., 54, 55 and older. This is followed by the ASFR values in 1948 for ages 12 and younger, 13, etc. While users of statistical packages are very familiar with this universal data format, users who work in Excel may find it difficult to operate with such data. For many purposes, it would be more useful to transform these data into a rectangular matrix of ASFRs, with columns corresponding to calendar years and rows corresponding to the mothers' ages at birth. The latter data shape can be readily used in Excel for constructing a figure with fertility age curves by year and various calculations.

Figure 3 presents another fragment of age-specific data from the HFD in the long format. These are cohort ASFRs by the mother's year of birth (cohort), the mother's age at birth, and birth order (1, 2, $3,4,5+)$. 


\begin{tabular}{|c|c|c|c|c|c|c|c|}
\hline & ASFR & ASFR1 & ASFR2 & ASFR3 & ASFR4 & ASFR 5 F \\
\hline \multicolumn{3}{|c|}{  } & - & - & - & - & . \\
\hline & - & - & - & - & . \\
\hline 1933 & 14 & 0.00153 & 0.00151 & 0.00002 & 0.00000 & 0.00000 & 0.00000 \\
\hline \multirow{2}{*}{$\begin{array}{l}1933 \\
1933\end{array}$} & 15 & 0.00570 & 0.00556 & 0.00013 & 0.00000 & 0.00000 & 0.00000 \\
\hline & 16 & 0.02110 & 0.02042 & 0.00057 & 0.00000 & 0.00000 & 0.00000 \\
\hline \multirow{2}{*}{$\begin{array}{l}1933 \\
1933\end{array}$} & 17 & 0.04585 & 0.04322 & 0.00253 & 0.00001 & 0.00000 & 0.00000 \\
\hline & 18 & 0.07557 & 0.06820 & 0.00799 & 0.00030 & 0.00007 & 0.00000 \\
\hline \multirow{2}{*}{1933} & 19 & 0.11371 & 0.09405 & 0.01685 & 0.00245 & 0.00033 & 0.00003 \\
\hline & 20 & 0.15565 & 0.12059 & 0.02958 & 0.00475 & 0.00051 & 0.0001 \\
\hline \multirow{2}{*}{$\begin{array}{l}1933 \\
1933\end{array}$} & 21 & 0.17565 & 0.12132 & 0.04546 & 0.00905 & 0.00070 & 0.00012 \\
\hline & 22 & 0.18849 & 0.11167 & 0.05224 & 0.01310 & 0.00147 & 0.00001 \\
\hline \multirow{2}{*}{$\begin{array}{l}1933 \\
1933\end{array}$} & 23 & 0.18380 & 0.08943 & 0.07305 & 0.01645 & 0.00445 & 0.00042 \\
\hline & 24 & 0.17334 & 0.06599 & 0.07852 & 0.01997 & 0.00631 & 0.00255 \\
\hline 1933 & 25 & 0.15625 & 0.04558 & 0.07782 & 0.02157 & 0.00779 & 0.00349 \\
\hline \multirow{3}{*}{$\begin{array}{l}1933 \\
1933 \\
1933\end{array}$} & 26 & 0.13414 & 0.03165 & 0.05874 & 0.02057 & 0.00841 & 0.0047 \\
\hline & 27 & 0.11895 & 0.02288 & 0.05244 & 0.01932 & 0.00858 & 0.00552 \\
\hline & 28 & 0.10017 & 0.01659 & 0.05182 & 0.01658 & 0.00832 & 0.0056 \\
\hline \multirow{2}{*}{$\begin{array}{l}1933 \\
1933\end{array}$} & 29 & 0.08472 & 0.01256 & 0.04223 & 0.01475 & 0.00773 & 0.00733 \\
\hline & 30 & 0.06963 & 0.00946 & 0.03308 & 0.01241 & 0.00694 & 0.0077 \\
\hline 1933 & 31 & 0.05333 & 0.00707 & 0.02333 & 0.00943 & 0.00567 & 0.0078 \\
\hline \multirow{2}{*}{$\begin{array}{l}1933 \\
1933\end{array}$} & 32 & 0.04182 & 0.00560 & 0.01656 & 0.00713 & 0.00438 & 0.0080 .5 \\
\hline & 33 & 0.03246 & 0.00445 & 0.01198 & 0.00527 & 0.00349 & 0.00727 \\
\hline 1933 & 34 & 0.02735 & 0.00365 & 0.00925 & 0.00518 & 0.00290 & 0.00637 \\
\hline \multirow{2}{*}{$\begin{array}{l}1933 \\
1933\end{array}$} & 35 & 0.02295 & 0.00294 & 0.00708 & 0.00530 & 0.00235 & 0.00529 \\
\hline & 36 & 0.01811 & 0.00233 & 0.00529 & 0.00421 & 0.00182 & 0.00446 \\
\hline 1933 & 37 & 0.01352 & 0.00172 & 0.00370 & 0.00288 & 0.00131 & 0.00390 \\
\hline \multirow{2}{*}{$\begin{array}{l}1933 \\
1933\end{array}$} & 38 & 0.00955 & 0.00139 & 0.00250 & 0.00187 & 0.00097 & 0.00292 \\
\hline & 39 & 0.00729 & 0.00115 & 0.00175 & 0.00128 & 0.00078 & 0.00233 \\
\hline 1933 & 40 & 0.00530 & 0.00083 & 0.00117 & 0.00089 & 0.00056 & 0.00185 \\
\hline & 41 & 0.00380 & 0.00053 & 0.00084 & 0.00060 & 0.00035 & 0.00147 \\
\hline 1933 & 42 & 0.00250 & 0.00035 & 0.00051 & 0.00035 & 0.00021 & 0.00108 \\
\hline 1933 & 43 & 0.00150 & 0.00023 & 0.00024 & 0.00023 & 0.00013 & 0.00068 \\
\hline 1933 & 44 & 0.00085 & 0.00013 & 0.00011 & 0.00017 & 0.00008 & 0.00038 \\
\hline 1933 & 45 & 0.00035 & 0.00005 & 0.00005 & 0.00005 & 0.00004 & 0.00015 \\
\hline 1933 & 46 & 0.00022 & 0.00003 & 0.00003 & 0.00004 & 0.00003 & 0.00010 \\
\hline 1933 & 47 & 0.00013 & 0.00003 & 0.00002 & 0.00002 & 0.00001 & 0.00000 \\
\hline 1933 & 48 & 0.00000 & 0.00000 & 0.00000 & 0.00000 & 0.00000 & 0.00000 \\
\hline 1933 & 49 & 0.00002 & 0.00001 & 0.00000 & 0.00000 & 0.00000 & 0.00000 \\
\hline 1933 & 50 & 0.00003 & 0.00002 & 0.00000 & 0.00000 & 0.00000 & 0.00000 \\
\hline 1933 & 51 & 0.00001 & 0.00001 & 0.00000 & 0.00000 & 0.00000 & 0.00000 \\
\hline 1933 & 52 & 0.00000 & 0.00000 & 0.00000 & 0.00000 & 0.00000 & 0.00000 \\
\hline 1933 & 53 & 0.00000 & 0.00000 & 0.00000 & 0.00000 & 0.00000 & 0.00000 \\
\hline 1933 & 54 & 0.00001 & 0.00000 & 0.00000 & 0.00000 & 0.00000 & 0.00000 \\
\hline 1933 & $55+$ & 0.00000 & 0.00000 & 0.00000 & 0.00000 & 0.00000 & 0.00000 \\
\hline 1934 & $13-$ & & - & - & - & - & . \\
\hline 1934 & 13 & 0.00009 & 0.00009 & 0.00000 & 0.00000 & 0.00000 & 0.00000 \\
\hline 1934 & 14 & 0.00164 & 0.00162 & 0.00002 & 0.00000 & 0.00000 & 0.00000 \\
\hline 1934 & 15 & 0.00724 & 0.00710 & 0.00014 & 0.00000 & 0.00000 & 0.00000 \\
\hline 1934 & 16 & 0.02138 & 0.02070 & 0.00058 & 0.00000 & 0.00000 & 0.00000 \\
\hline 1934 & 17 & 0.04502 & 0.04222 & 0.00278 & 0.00001 & 0.00000 & 0.00000 \\
\hline 1934 & 18 & 0.07804 & 0.05953 & 0.00810 & 0.00027 & 0.00003 & 0.00000 \\
\hline 1934 & 19 & 0.11795 & 0.09863 & 0.01651 & 0.00249 & 0.00027 & 0.0000 \\
\hline 1934 & 20 & 0.15922 & 0.12357 & 0.03022 & 0.00504 & 0.00034 & 0.00005 \\
\hline
\end{tabular}

Figure 3. Cohort age-specific fertility rates by the year of the mother's birth, the age of the mother, and birth order. A fragment of HFD data for Bulgaria.

Such HFD data can be transformed into five rectangular (age)x(year) matrices corresponding to birth orders 1, 2, 3, 4, 5, and higher.

This technical report provides an Excel spreadsheet for reshaping long-format data into wide format.

\section{How to use it}

The "Reshape-Wide_2.xls" spreadsheet has two worksheets: "ReadMe" with a short explanatory note, and "InputData." The input (long) HFD data should be placed in the green range on the InputData worksheet.

This can be done in several ways. For example, on the HFD Age-Specific Data webpage of the country of interest, you can click on the dataset that you want to reshape. The selected file will appear on the screen. Select the entire range of ASFRs (you may press Ctrl-A to select all data) and press Ctrl-C for copying. 
Open a blank spreadsheet in Excel and paste the data in it by clicking on the Paste button (Home tab in Office-2007) or by pressing Ctrl-V. Split the data by column using the "DatalText to Columns" menu (Data tab\Text to column button). When splitting the text by column, you can select "space" as the column delimiter. Select the data range and copy it to the green range in the InputData worksheet.

Press the green button in the InputData worksheet to transform data into wide format. The output wide data will appear in a newly opened spreadsheet, which will be activated. This spreadsheet can then be saved in any folder.

Go back to the InputData page in Reshape-Wide_2.xls and press the yellow button to clean the input range.

\section{References}

HFD 2012. The Human Fertility Database. Available at http://www.humanfertility.org 